\title{
Fli1-haploinsufficient dermal fibroblasts promote skin-localized transdifferentiation of Th2-like regulatory T cells
}

\author{
Ryosuke Saigusa', Yoshihide Asano ${ }^{1 *}$, Takashi Taniguchi', Megumi Hirabayashi', Kouki Nakamura', \\ Shunsuke Miura', Takashi Yamashita', Takehiro Takahashi', Yohei Ichimura', Tetsuo Toyama', Ayumi Yoshizaki', \\ Maria Trojanowska ${ }^{2}$ and Shinichi Sato ${ }^{1}$
}

\begin{abstract}
Background: Friend leukemia virus integration 1 (Fli1) deficiency, a predisposing factor of systemic sclerosis (SSC), induces SSc-like phenotypes in various cell types. A recent study demonstrated the transdifferentiation of T helper type 2 cell (Th2)-like regulatory T cells (Tregs) in SSc lesional skin through interleukin (IL)-33 produced by fibroblasts. Therefore, we investigated the role of Fli1 deficiency in dermal fibroblast-mediated transdifferentiation of Tregs.

Methods: Cytokine expression was assessed in Tregs by flow cytometry and in skin samples and cultivated cells by immunostaining, immunoblotting, and/or qRT-PCR. Fli1 binding to the target gene promoters was examined by chromatin immunoprecipitation. Murine dermal fibroblasts and Tregs were cocultured with or without blocking antibodies against target cytokines.

Results: Th2- and Th17-like cell proportions in skin-homing Tregs were increased in bleomycin-treated Fli ${ }^{+/-}$mice compared with bleomycin-treated wild-type mice, whereas Th1-, Th2-, and Th17-like cell proportions in splenic Tregs were comparable. Fli ${ }^{+/-}$fibroblasts overproduced IL-33 and IL-6, in particular IL-33, and Fli1 occupied the IL33 and IL6 promoters in dermal fibroblasts. Importantly, the IL-4-producing cell proportion was significantly higher in wild-type Tregs cocultured with $\mathrm{Fli}^{+/-}$fibroblasts than in those cocultured with wild-type fibroblasts, which were canceled by neutralizing anti-IL-33 antibody. Under the same coculture condition, an increased tendency of IL-17A-producing cell proportion, which was possibly mediated by IL-6, was evident.

Conclusions: Fli1 haploinsufficiency increases the proportions of Th2- and Th17-like Tregs in bleomycin-induced profibrotic skin conditions, in which IL-33-producing dermal fibroblasts contribute to Th2-like Treg transdifferentiation, suggesting a critical role of Fli1 deficiency in the interaction of dermal fibroblasts with immune cells in pathological skin fibrosis.
\end{abstract}

Keywords: Fli1, Fibroblasts, Regulatory T cells, IL-33, Systemic sclerosis

\section{Background}

Systemic sclerosis (SSc) is a multisystem autoimmune disease characterized by vasculopathy and fibrosis of the skin and certain internal organs. Although SSc pathogenesis has yet to be fully disclosed, the canonical wisdom is that dermal fibroblast activation is the final consequence of its sequential pathological processes, such as initial vascular injury due to autoimmune attack and subsequent chronic

\footnotetext{
* Correspondence: yasano-tky@umin.ac.jp

'Department of Dermatology, University of Tokyo Graduate School of Medicine, 7-3-1 Hongo, Bunkyo-ku, Tokyo 113-8655, Japan

Full list of author information is available at the end of the article
}

inflammation [1]. However, a recent study has demonstrated that activated fibroblasts regulate tissue-localized transdifferentiation of regulatory $\mathrm{T}$ cells (Tregs) into $\mathrm{T}$ helper type 2 cell (Th2)-like cells through IL-33 in SSc lesional skin [2], suggesting that activated dermal fibroblasts amplify an aberrant immune response characteristic of SSc. This notion provides new insight into the continuing discussion of the controversy of Tregs in SSc, with reports of decreased, increased, or equal proportions of Tregs in the blood of patients compared with healthy control subjects [3-6]. Although Treg transdifferentiation into Th1- and/or Th17-like cells has been reported in type 1 
diabetes [7], multiple sclerosis [8], and juvenile idiopathic arthritis [9], Treg transdifferentiation into Th2-like cells is documented exclusively in SSc so far, indicating that this pathological process is unique in this disease.

Friend leukemia virus integration 1 (Fli1) is a member of the Ets family of transcription factors, the deficiency of which is a potential predisposing factor of SSc [10]. Fli1 expression is suppressed in dermal fibroblasts, endothelial cells, and perivascular inflammatory cells in involved and noninvolved skin of patients with SSc [11]. Although the detailed mechanism explaining Fli1 downregulation in SSc is still unknown, an epigenetic mechanism is reported at least in dermal fibroblasts [12]. According to a series of studies on Fli1-mutated mice and FLI1 small interfering RNA-treated cultured cells, Fli1 deficiency promotes the induction of SSc-like phenotypes in dermal fibroblasts, dermal microvascular endothelial cells, and macrophages [13-16]. Most importantly, mice with simultaneous haploinsufficiency of the Fli1 and Klf5 genes, both of which are epigenetically suppressed in SSc dermal fibroblasts, spontaneously develop the three cardinal features of SSc, including immune abnormalities, vasculopathy, and tissue fibrosis [17]. These animal models are useful for obtaining a clue to understanding the role of certain cells and to elucidating the mechanisms of disease-modifying drugs in SSc $[18,19]$.

On the basis of this background, we investigated the role of Fli1 deficiency in fibroblast-mediated transdifferentiation of Tregs by using bleomycin (BLM)-treated $\mathrm{Fli}^{+/-}$mice. The detailed molecular mechanism was further investigated by coculture experiments of Tregs with dermal fibroblasts.

\section{Methods}

\section{A BLM-induced murine SSc model}

BLM (200 g, 02907278; Nippon Kayaku, Tokyo, Japan) dissolved in PBS or control PBS was injected subcutaneously into a single location on the back of 8-week-old female wild-type (WT) mice (C57BL/6) and $\mathrm{Fli1}^{+/-}$mice daily.

\section{Flow cytometry}

Mice were treated with BLM for 1 week. On the day after the final injection, lymphocytes from the spleen and the dermis of the lower back skin were obtained. Skin samples were incubated in $2 \mathrm{mg} / \mathrm{ml}$ dispase (383-02281; Wako Pure Chemical Industries, Osaka, Japan) and were separated into epidermis and dermis. Dermis was minced and then incubated with $2 \mathrm{mg} / \mathrm{ml}$ collagenase type 2 (CLS-2; Worthington Biochemical, Lakewood, NJ, USA) in Tyrode's solution for 60-90 minutes. The digested tissues were centrifuged, resuspended in PBS, and filtered through a $70-\mu \mathrm{m}$ mesh. Single-cell suspensions were stained on ice with labeled monoclonal antibody. In the surface staining experiments, cells were stained with antibodies against CD4 (100526; BioLegend, San Diego, CA, USA). In intracellular cytokine staining, cells were stimulated with $10 \mathrm{ng} / \mathrm{ml}$ phorbol myristate acetate and $1 \mu \mathrm{g} / \mathrm{ml}$ ionomycin (10634; SigmaAldrich, St. Louis, MO, USA) in the presence of $1 \mathrm{mg} / \mathrm{ml}$ brefeldin A (420601; BioLegend) for $4 \mathrm{~h}$. Cells were washed; stained for CD4; treated with fixative/permeabilization buffer (71-5775-40; eBioscience, San Diego, CA, USA); and then stained with anti-FoxP3 (71-5775-40; eBioscience), anti-IL-4 (554436; BD Biosciences, San Jose, CA, USA), anti-IL-13 (12-7133-41; eBioscience), anti-IL-17A (506919; BioLegend), and anti-interferon (IFN)- $\gamma$ (505825; BioLegend) antibodies. Tregs were defined by $\mathrm{CD} 4^{+} \mathrm{FoxP}^{+}$cells. Cells were analyzed on a FACSVerse flow cytometer (BD Biosciences).

\section{Immunohistochemistry}

Immunohistochemistry with the Mouse on Mouse $\left(\right.$ M.O.M ${ }^{\mathrm{TM}}$ ) Elite Peroxidase Detection Kit (PK-2200; Vector Laboratories, Burlingame, CA, USA) was performed on formalin-fixed, paraffin-embedded skin sections using anti-IL-33 antibody (ab54384; Abcam, Cambridge, UK). To quantify the signal intensity of IL33, color images were converted to grayscale, and then the brightness was measured in five different randomly selected fibroblasts and epidermal areas per specimen. These processes were analyzed using ImageJ software (National Institutes of Health, Bethesda, MD, USA), and the average values were compared.

\section{Murine dermal fibroblast isolation}

For isolation of murine fibroblasts, referring to a past report [20], we took the ears from mice and placed them in cell culture media. Then, collagenase (17100017; Thermo Fisher Scientific, Waltham, MA, USA) was added, and the ears were minced as much as possible with sterile scissors. After overnight incubation at $37^{\circ} \mathrm{C}$, the ears were further dissociated by vigorous pipetting. Cells were passed through a cell strainer to achieve a single-cell suspension and remove the remaining debris. Finally, the cells were pelleted by centrifugation, resuspended, and cultured until proliferated.

\section{qRT-PCR}

RNA isolation from cultivated cells and skin tissue, as well as qRT-PCR, was performed as described previously [21-23]. The sequences of primers were as follows: murine Il33-forward 5'-CAATCAGGCGACG GTGTGGATGG-3', murine Il33-reverse 5'-TCCGGA GGCGAGACGTCACC-3'; murine Il6-forward 5'-GA TGGATGCTACCAAACTGGAT-3', murine Il6-reverse 5'-CCAGGTAGCTATGGTACTCCAGA-3'; murine Gapdh-forward 5'-CGTGTTCCTACCCCCAATGT-3', murine Gapdh-reverse 5'-TGTCATCATACTTGGCA GGTTTCT-3'. 


\section{Immunoblotting}

Confluent quiescent fibroblasts were serum-starved for $24 \mathrm{~h}$. Whole-cell lysates were prepared. Samples were subjected to sodium dodecyl sulfate-PAGE (NP0321; Life Technologies/Thermo Fisher Scientific, Carlsbad, CA, USA) and immunoblotting with anti-IL-33 antibody (ab54384; Abcam) and anti- $\beta$-actin antibody (sc-47778; Santa Cruz Biotechnology, Dallas, TX, USA). Bands were detected using enhanced chemiluminescence techniques (34080; Thermo Fisher Scientific, Rockford, IL, USA).

\section{Chromatin immunoprecipitation assay}

Human normal dermal fibroblasts were purchased from the American Type Culture Collection (PCS-201-010; Manassas, VA, USA) and used. The chromatin immunoprecipitation (ChIP) assay was conducted using the EpiQuik ChIP kit (P-2002-3; EpiGentek, Farmingdale, NY, USA) [17]. Putative Fli1 binding sites in the IL33 and IL6 promoters were predicted using Tfsitescan. The primers that amplify fragments of the IL33 promoter ( -1332 bp to approximately $-1183 \mathrm{bp}$ ) and the IL6 promoter (-1109 bp to approximately $-944 \mathrm{bp})$ were as follows: IL33 ChIPforward 5'-TCAGCTGGGAGATGGGTAAG-3'; IL33 ChIP-reverse 5' ${ }^{\prime}$-ATAATCTATTCTCTCTGAAGCCTACAA-3', IL6 ChIP-forward 5'-GACACCATCCTGAG GGAAGA-3'; IL6 ChIP-reverse 5'-TATCGC TCCCTCTCCCTGTA-3'. In some experiments, fibroblasts were treated with IL-1 $\beta$ (201-LB-005; R\&D Systems, Minneapolis, MN, USA).

\section{Cocultures}

Murine dermal fibroblasts were prepared from WT and $\mathrm{Fli1}^{+/-}$mice and maintained as described previously [13]. Splenic Tregs were isolated from WT mice with a CD4 ${ }^{+}$CD25 ${ }^{+}$Treg cell isolation kit (130-091-041; Miltenyi Biotec, Bergisch Gladbach, Germany) and cultured in RPMI 1640 medium supplemented with FCS. Murine dermal fibroblasts $\left(1 \times 10^{5}\right.$ cells $)$ and $\mathrm{CD} 4^{+} \mathrm{CD} 25^{+} \mathrm{T}$ cells $\left(3 \times 10^{5}\right.$ cells) were cocultured in 24-well plates for 2 days. Then, cells were analyzed on a FACSVerse flow cytometer. In some experiments, cocultured cells were treated with anti-mouse IL-33 antibody (M187-3; MBL, Nagoya, Japan) or antimouse IL-6 antibody (MAB406-SP; R\&D Systems).

\section{Statistical analysis}

Statistical analysis was done with the Mann-Whitney $U$ test to compare the distributions of two unmatched groups. Statistical significance was defined as a $P$ value $<0.05$.

\section{Results}

Th2- and Th17-like Tregs are increased in the skin of BLM-treated $\mathrm{Fli}^{+/-}$mice

As an initial experiment, we employed BLM-treated mice because this murine model recapitulates inflammatory and fibrotic aspects of SSc [24]. In this model, inflammation and dermal fibrosis reach their peak at days 7 and 28 after BLM injection, respectively [25-28]. After confirming that BLM induces greater dermal fibrosis in $\mathrm{Flil}^{+/-}$ mice than in WT mice as previously reported [13], we collected skin samples and splenocytes at day 7 and evaluated the phenotypes of skin-homing and splenic Tregs by comparing the proportions of IFN- $\gamma^{-}$, IL-4-, IL-13-, and IL-17A-producing cells among $\mathrm{CD}^{+}{ }^{+} \mathrm{FoxP}^{+}$cells. As shown in Fig. 1a, on one hand, BLM injection increased the proportions of IL-4-, IL-13-, and IL-17A-producing Tregs-namely, Th2- and Th17-like Tregs-in the skin of $\mathrm{Fli1}^{+/-}$mice compared with WT mice. On the other hand, the proportions of Th1-, Th2-, and Th17-like cells in splenic Tregs were comparable between BLM-treated Fli1 +/- mice and BLM-treated WT mice (Fig. 1b; scatterplots also shown in Additional file 1: Figure S1). These results suggest that Fli1 haploinsufficiency promotes skinlocalized transdifferentiation and/or skin infiltration of Th2- and Th17-like Tregs in BLM-treated mice.

\section{IL-33 expression is higher in skin of BLM-treated $\mathrm{Fli1}^{+/-}$ mice}

Because IL-33 produced by dermal fibroblasts contributes to Th2-like Treg transdifferentiation in the lesional skin of patients with SSc [2], we next evaluated IL-33 expression in the skin of BLM-treated $\mathrm{Fli1}^{+/-}$mice. Of note, Il33 messenger RNA (mRNA) expression was higher in the skin of BLM-treated $\mathrm{Fli1}^{+/-}$mice than in BLM-treated WT mice (Fig. 1c). To confirm the origin of BLM-induced IL-33 expression, we also carried out immunostaining with anti-IL-33 antibody. As shown in Fig. 1d and Additional file 2: Figure S2, the signal intensity of IL-33 in dermal fibroblasts was remarkably increased in BLM-treated $\mathrm{Flil}^{+/-}$mice as compared with BLM-treated WT mice, whereas that in epidermal keratinocytes was comparable. When the staining was performed without anti-IL-33 antibody, no signals were detectable in the same skin sample from BLM-treated WT mice (Fig. 1e). Taken together, these results indicate that IL-33 produced by dermal fibroblasts may contribute to Th2-like Treg transdifferentiation in the skin of BLM-treated $\mathrm{Flil}^{+/-}$mice.

Fli1 haploinsufficiency results in overexpression of IL-33 in dermal fibroblasts

To investigate the molecular mechanism underlying IL33 overproduction in dermal fibroblasts of BLM-treated $\mathrm{Fli1}^{+/-}$mice, we first evaluated IL-33 expression levels in the skin of $\mathrm{Fli1}^{+/-}$and WT mice under a physiological condition. Of note, Il33 mRNA levels were significantly elevated in the skin of $\mathrm{Fli1}^{+/-}$mice compared with WT mice (Fig. 2a). Furthermore, IL-33 expression was clearly elevated in dermal fibroblasts of $\mathrm{Fli1}^{+/-}$mice relative to 


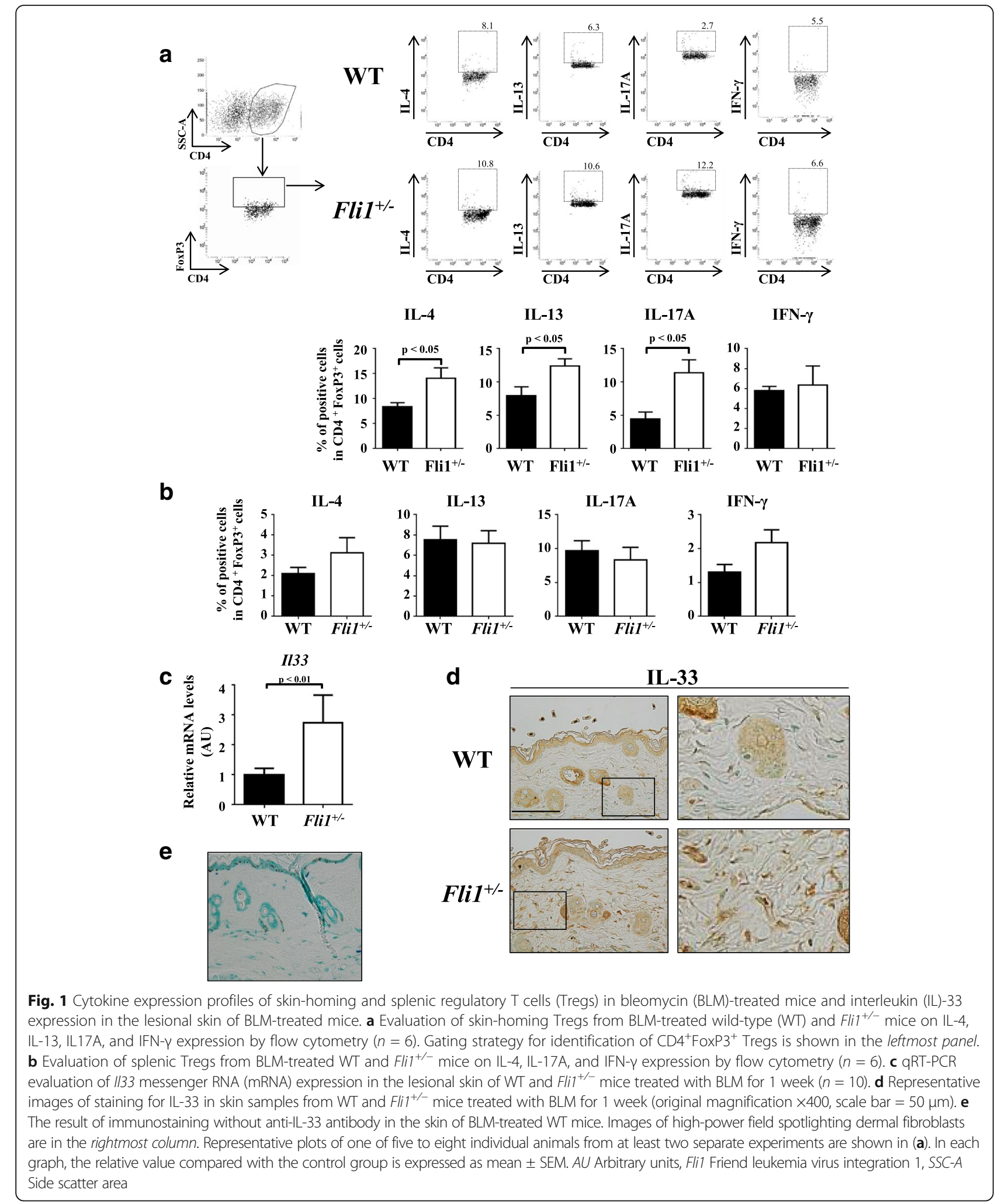

those cells of WT mice while being comparable in epidermal keratinocytes (Fig. 2b and Additional file 2: Figure S2). Also, no nonspecific signals were confirmed by conducting staining without anti-IL-33 antibody (Fig. 2c). Importantly, the signal intensity of IL-33 in dermal fibroblasts was modestly but significantly elevated in BLM-treated $\mathrm{Fli1}^{+/-}$mice 

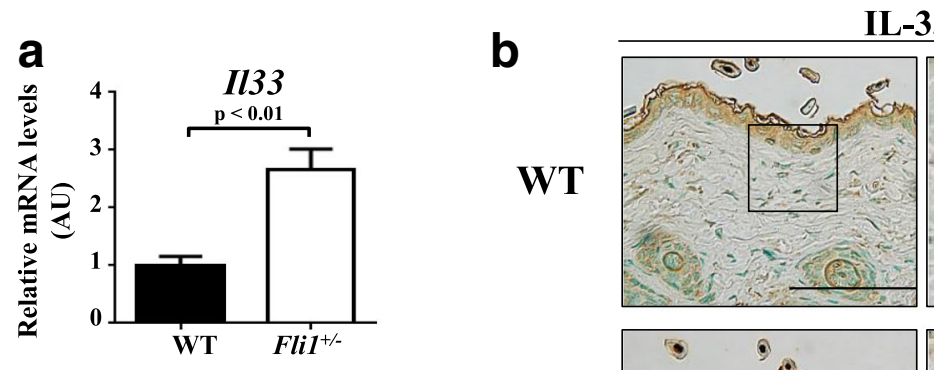

IL-33

C
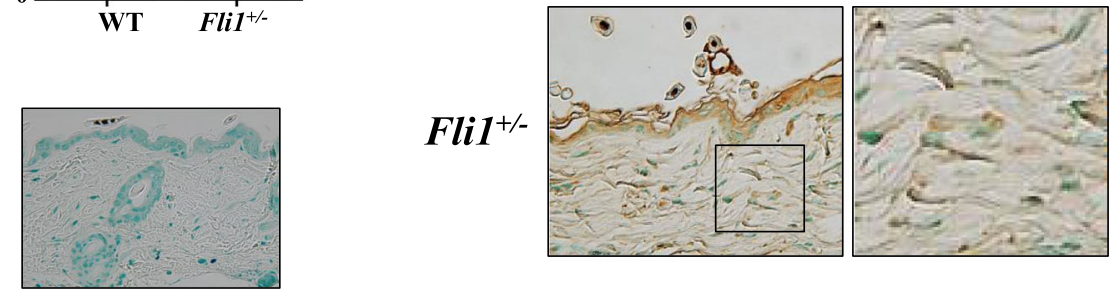

d

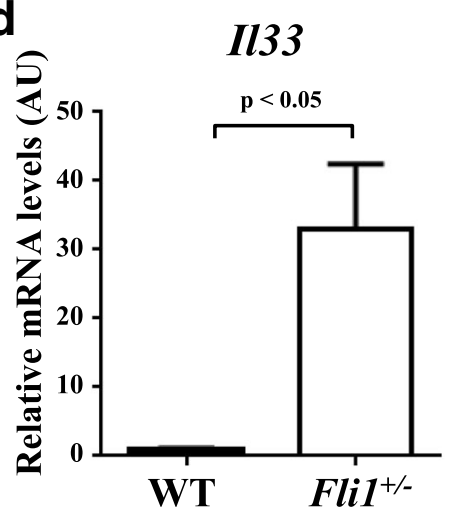

e

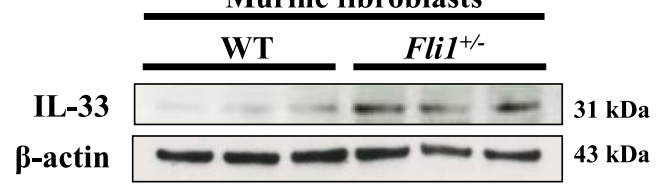

$\mathbf{g}$

Human fibroblasts
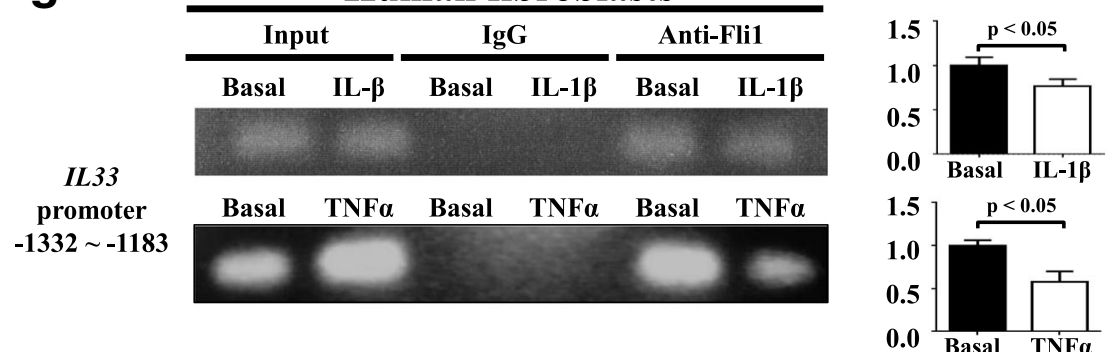

Fig. 2 Friend leukemia virus integration 1 (Fli1) regulates the expression of interleukin (IL)-33 in dermal fibroblasts. a, b Evaluation of IL-33 expression in skin from wild-type $(\mathrm{WT})$ and Fli ${ }^{+/-}$mice without any treatment by qRT-PCR $(\mathbf{a}, n=10)$ and immunohistochemistry $(\mathbf{b}, n=5$; original magnification $\times 400$, scale bar $=50 \mu \mathrm{m})$. High-power field images spotlighting dermal fibroblasts are in the rightmost column. $\mathbf{c}$ The result of immunostaining without anti-IL-33 antibody in the skin of WT mice under a physiological condition. d, e Evaluation of IL-33 expression in dermal fibroblasts isolated from WT and $\mathrm{Fli}^{+/-}$mice by qRT-PCR $(\mathbf{d}, n=10)$ and immunoblotting $(\mathbf{e}, n=4)$. $\mathbf{f}, \mathbf{g}$ Results of the chromatin immunoprecipitation assay regarding Fli1 occupation on the IL33 promoter in human dermal fibroblasts without any treatment $(\mathbf{f}, n=4)$ and those cells treated with IL-1 $\beta$ or tumor necrosis factor (TNF)-a for 24 $h(\mathbf{g}, n=4)$. For $\mathbf{a}$ and $\mathbf{c}$, messenger RNA (mRNA) levels of the $/ / 33$ gene were normalized to those of the Gapdh gene. For $\mathbf{f}$, the occupancy of the $/ / 33$ promoter by Fli1 was quantified with qRT-PCR. For $\mathbf{b}, \mathbf{d}$, and $\mathbf{e}$, representative results are shown. In each graph, the relative value compared with the control group is expressed as mean \pm SEM. AU Arbitrary units, IgG Immunoglobulin G, TNF-a Tumor necrosis factor-a

compared with untreated $\mathrm{Fli1}^{+/-}$mice (Additional file 2: Figure S2). Supporting these in vivo findings, IL-33 expression was increased in $\mathrm{Flil}^{+/-}$dermal fibroblasts at mRNA and protein levels compared with WT dermal fibroblasts in vitro (Fig. 2d and e; see Additional file 3: Figure S3 for an original file). These results indicate that $\mathrm{Flil}^{+/-}$dermal fibroblasts produce excessive amounts of IL-33 even under a quiescent condition.

To further examine if Fli1 directly regulates the expression of IL-33, we conducted in vitro experiments with human dermal fibroblasts. The ChIP assay exhibited the binding of Fli1 to the IL33 promoter (Fig. 2f), 
suggesting the direct regulation of IL-33 by Fli1. Given that IL-1 $\beta$ and tumor necrosis factor (TNF)- $\alpha$ induce the expression of IL-33 in dermal fibroblasts [29], we investigated whether these molecules affect the occupancy of Fli1 on the IL33 promoter by performing ChIP analysis, showing that both of IL- $1 \beta$ and TNF- $\alpha$ induced the dissociation of Fli1 from the promoter (Fig. 2g). In aggregate, these results indicate that Fli1 directly regulates IL-33 expression as a potent repressor and that Fli1 deficiency possibly contributes to IL-33 production in SSc dermal fibroblasts.

\section{Transdifferentiation of Tregs into Th2-like cells is pro-} moted by $\mathrm{Fli}^{+/-}$dermal fibroblasts through IL-33 in vitro Because fibroblast-mediated transdifferentiation of Th2like Tregs depends on IL-33 in SSc, we next assessed if the same scenario is applicable to Fli1 haploinsufficiencydependent transdifferentiation of Th2-like Tregs in BLMtreated mice. To this end, we cocultured WT Tregs with dermal fibroblasts isolated from WT mice or $\mathrm{Fli1}^{+/-}$mice. The proportion of IL-4-producing cells was significantly higher and the proportion of IL-17A-producing cells tended to be higher in $\mathrm{CD} 4^{+} \mathrm{FoxP}^{+}$cells cocultured with $\mathrm{Fli1}^{+/-}$dermal fibroblasts than in those cells cocultured with WT fibroblasts, whereas no effect was seen on the proportion of IFN- $\gamma$-producing cells (Fig. 3a). On the basis of the data up to this point, Fli1 deficiency in dermal fibroblasts may induce IL-4-expressing Tregs, possibly through the key molecule IL-33. To address this issue, we cocultured these cells in the presence of neutralizing antibody for IL-33. As expected, this neutralizing antibody significantly attenuated the proportion of IL-4-producing cells, whereas it did not affect the proportions of IL-17Aand IFN- $\gamma$-producing cells in $\mathrm{CD}^{+}{ }^{+} \mathrm{FoxP}^{+}$cells cocultured with $\mathrm{Fli1}^{+/-}$dermal fibroblasts (Fig. 3b). These results indicate that $\mathrm{Fli1}^{+/-}$dermal fibroblasts regulate the transdifferentiation of Th2-like Tregs at least partially through IL-33.

We also assessed whether $\mathrm{Fli1}^{+/-}$dermal fibroblasts promote the transdifferentiation of Th17-like Tregs, because a significant increase in the proportion of IL-17A-producing Tregs in the skin of BLM-treated $\mathrm{Fli1}^{+/-}$mice (Fig. 1a) and an increased tendency of IL-17A-producing cells in WT Tregs cocultured with $\mathrm{Fli1}^{+/-}$dermal fibroblasts (Fig. 3a) were evident. Because IL- 6 is a key cytokine in the conversion of induced Tregs into Th17 cells $[30,31]$ and its mRNA expression is increased in the lesional skin of BLMtreated $\mathrm{Fli1}^{+/-}$mice compared with BLM-treated WT mice [13], we speculated that IL-6 produced by $\mathrm{Fli1}^{+/-}$dermal fibroblasts promotes the transdifferentiation of Th17-like Tregs. Consistent with this hypothesis, Il6 mRNA expression was significantly higher in $\mathrm{Fli1}^{+/-}$dermal fibroblasts than in WT dermal fibroblasts (Fig. 4a, left panel). Also, IL6 protein expression was elevated in cultured dermal fibroblasts from BLM-treated $\mathrm{Fli}^{+/-}$mice compared with those from BLM-treated WT mice (Fig. 4a, right panel; see Additional file 3: Figure S3 for an original file). The ChIP analysis revealed the binding of Fli1 to the IL6 promoter in human normal dermal fibroblasts, suggesting the direct regulation of IL-6 expression by Fli1 (Fig. 4b). More importantly, anti-IL- 6 antibody tended to reduce the proportion of Th17-like cells in WT Tregs cocultured with $\mathrm{Fli1}^{+/-}$dermal fibroblasts (Fig. 4c). Taken together, these results indicate that Fli1 haploinsufficiency increases the proportion of Th17-like cells in skin-homing Tregs, possibly and partially through IL- 6 produced by dermal fibroblasts.

\section{Discussion}

BLM-treated mice have been widely used as a useful model of SSc $[32,33]$. On one hand, the benefit of this model is the highly reproducible induction of inflammation and tissue fibrosis similar to those of SSc. In addition, it can be used in various genetically manipulated mice to explore the relationship of targeted molecules with inflammatory and fibrotic pathways related to SSc development. On the other hand, the weak point of this model is the lack of persistent tissue fibrosis like that in SSc; namely, tissue fibrosis spontaneously improves within 3 months after ceasing BLM injection. We currently focused on the contribution of Fli1 haploinsufficiency to dermal fibroblast-dependent transdifferentiation of Tregs during the inflammatory phase of an SSc-like condition; therefore, we can say that BLM-treated mice would be suitable for addressing this issue. In our initial experiments, the proportions of Th2- and Th17-like cells in skin-homing Tregs were significantly increased in BLM-treated $\mathrm{Fli1}^{+/-}$ mice compared with BLM-treated WT mice, which is consistent with our previous report demonstrating the increased expression of Th2 and Th17 cytokines in the lesional skin of BLM-treated mice [13]. Importantly, Th2like Treg transdifferentiation was regulated at least partially by IL-33 overproduced by $\mathrm{Fli1}^{+/}$dermal fibroblasts. In addition, IL- 6 produced by $\mathrm{Fli1}^{+/-}$dermal fibroblasts seemed to be modestly involved in Th17-like Treg transdifferentiation. Because Fli1 occupied the IL33 and IL6 promoters, Fli1 haploinsufficiency is likely to directly enhance the production of IL-33 and IL- 6 in dermal fibroblasts. These results indicate that Fli1 haploinsufficiency augments BLM-induced skin fibrosis by increasing the production of profibrotic Th2 cytokines and the induction of Th17 cytokine-mediated inflammation partially through the interaction of dermal fibroblasts and Tregs.

A critical contribution of Fli1 deficiency to the activation of dermal fibroblasts was initially proved on the basis of its impact on type I collagen expression. Fli1 binds to the COL1A1 and COL1A2 promoters [21, 34] and serves as a potent repressor of these genes in dermal 

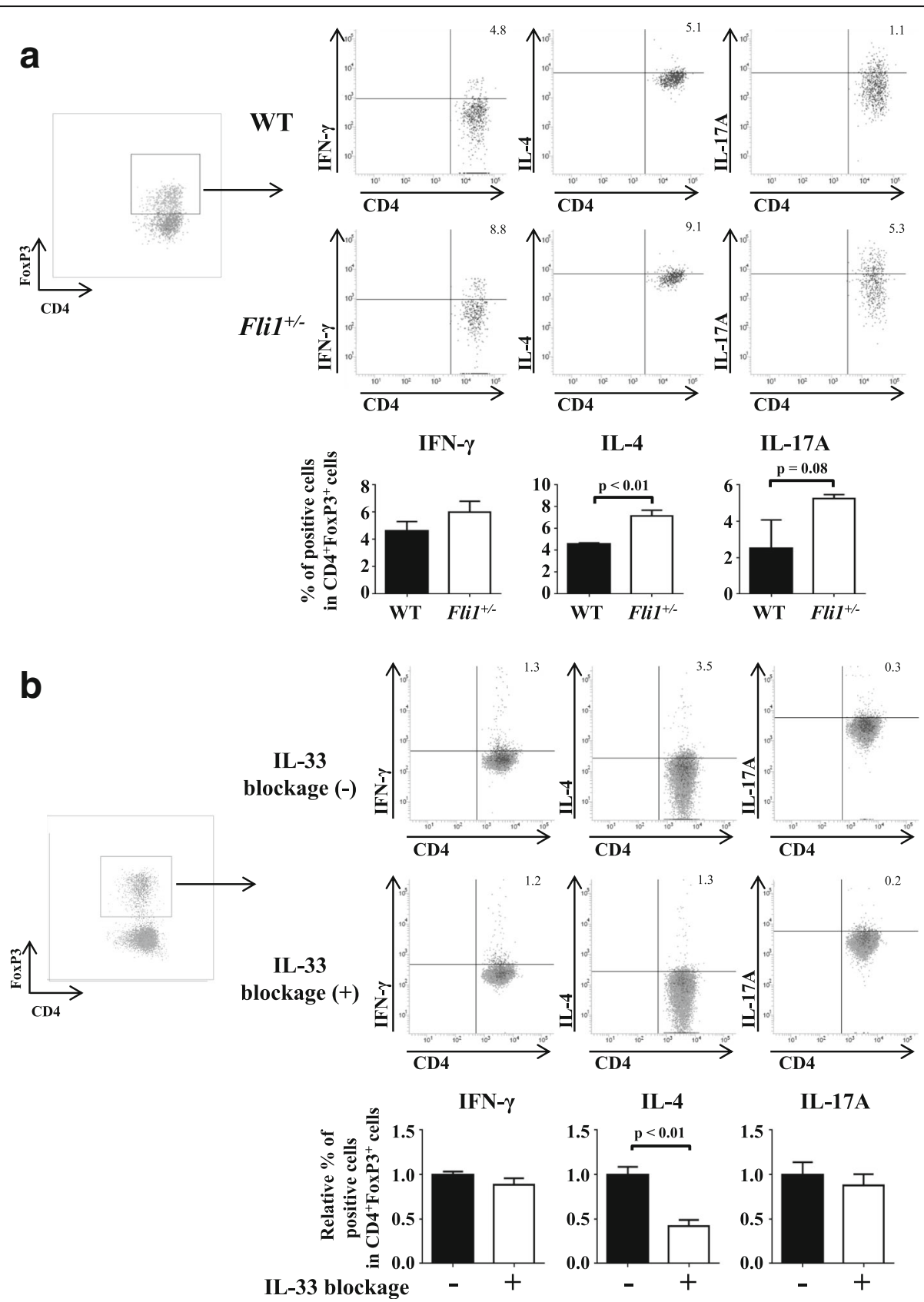

Fig. 3 T helper type 2 cell (Th2)-like regulatory T cells (Tregs) are induced by coculture with $\mathrm{Fli}^{+/-}$dermal fibroblasts through interleukin (IL)-33. a Evaluation by flow cytometry of Th1-, Th2-, and Th17-like Treg induction by coculture with wild-type (WT) and Fli ${ }^{+/-}$dermal fibroblasts $(n=6)$. $\mathbf{b}$ Evaluation by flow cytometry of the effect of IL-33-neutralizing antibody on Tregs cocultured with Fli ${ }^{+/-}$dermal fibroblasts $(n=6)$. Representative plots of interferon (IFN)- $\gamma^{-}$, IL-4-, and IL-17A-positive Tregs are shown in right upper panels of (a) and (b). Gating strategy for identification of CD4 ${ }^{+}$FoxP $3^{+}$Tregs is shown in the leftmost panels of (a) and (b). In each graph, the relative value compared with the control group is expressed as mean \pm SEM. AU Arbitrary units, Fli1 Friend leukemia virus integration 1

fibroblasts [35]. The inverse correlation between Fli1 and type I collagen expression is clearly shown in Fli1 ${ }^{+/+}, \mathrm{Fli1}^{+/-}$, and $\mathrm{Fli1}^{-/-}$murine embryonic fibroblasts [11]. In addition to type I collagen, Fli1 deficiency increases integrin $\alpha_{\mathrm{v}} \beta_{3}$ and $\alpha_{\mathrm{v}} \beta_{5}$ expression, establishing autocrine transforming growth factor (TGF) $\beta$ signaling in dermal fibroblasts [13]. Furthermore, Fli1 deficiency enhances progranulin production, which renders dermal fibroblasts resistant to the antifibrotic effect of TNF- $\alpha$ [36]. In the present study, we proposed a novel notion that Fli1 deficiency regulates skin-localized transdifferentiation of Th2-like Tregs through IL-33 and possibly that 
a

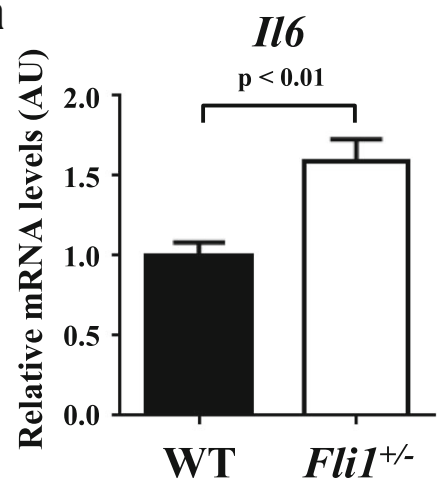

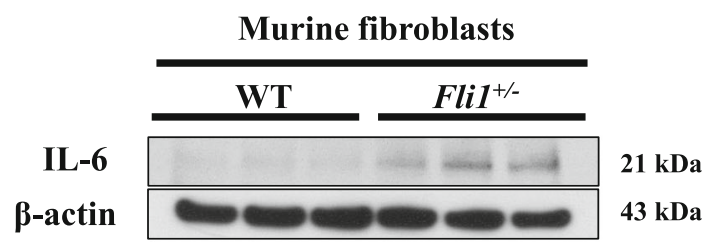

b

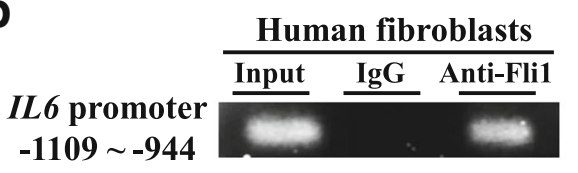

C
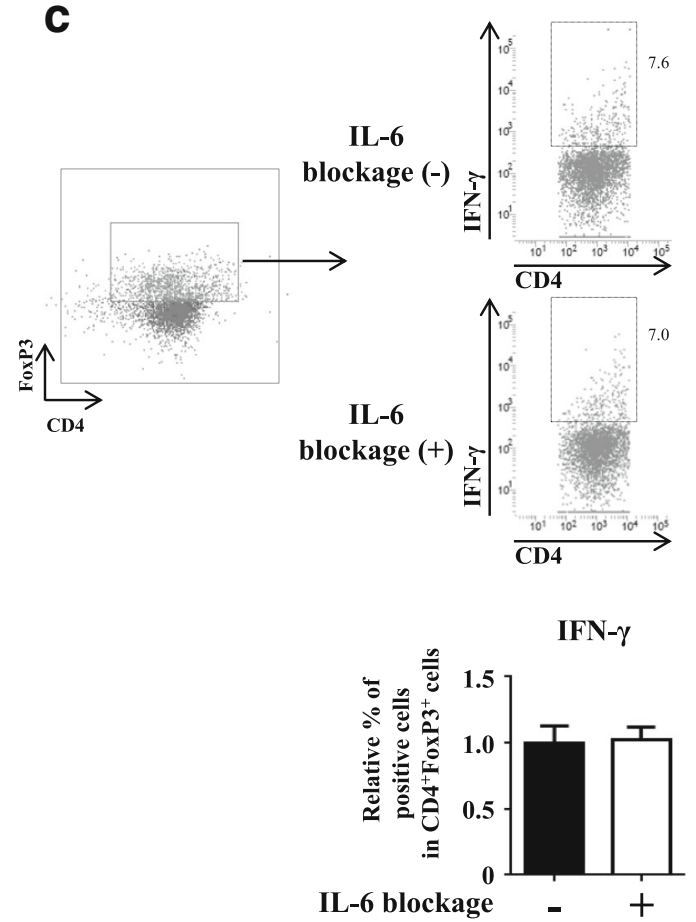
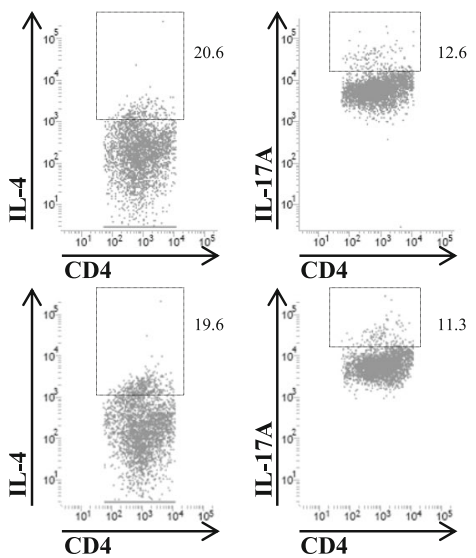

IL-4

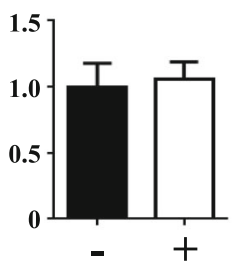

IL-17A

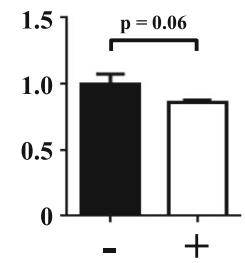

Fig. $4 \mathrm{Fli}^{+/-}$dermal fibroblasts may modestly and partially contribute to the induction of T helper type 17 cell (Th17)-like regulatory T cells (Tregs) by interleukin (IL)-6 production. a Evaluation of IL-6 expression in dermal fibroblasts isolated from wild-type (WT) and Fli ${ }^{+/-}$mice ( $n=10$ for each group) by qRT-PCR (left panel) and in dermal fibroblasts isolated from bleomycin-treated WT and Fli ${ }^{+/-}$mice ( $n=3$ for each group) by immunoblotting (right panel). $\mathbf{b}$ Results of the chromatin immunoprecipitation assay regarding Fli1 occupation on the $/ L 6$ promoter with human dermal fibroblasts $(n=4)$. $\mathbf{C}$ Evaluation by flow cytometry of the effect of IL-6 neutralizing antibody on Tregs cocultured with Fli ${ }^{+/-}$dermal fibroblasts $(n=6)$. Representative results of immunoblotting, agarose gel electrophoresis, and flow cytometry are shown for the right panels of $\mathbf{a}$ and $\mathbf{b}$ and the right upper panel of $\mathbf{c}$, respectively. Gating strategy for identification of $\mathrm{CD}^{+} \mathrm{FoxP3}^{+}$Tregs is shown in the leftmost panel of $\mathbf{c}$. In each graph, the relative value compared with the control group is expressed as mean \pm SEM. AU Arbitrary units, Fli1 Friend leukemia virus integration 1, IgG Immunoglobulin G, mRNA Messenger RNA

of Th17-like Tregs through IL-6, both of which are produced by dermal fibroblasts. Considering that Fli1 deficiency promotes endothelial-to-mesenchymal transition and M2 macrophage differentiation [13], Fli1 deficiency integrates fibrosis-related gene programs toward the profibrotic condition in various types of cells.

Th2-skewed immune polarization has been implicated in the development of SSc-associated tissue fibrosis on the basis of clinical data. In the early stage of diffuse cutaneous systemic sclerosis (dcSSc), serum IL-6 and IL-
10 levels are significantly elevated, whereas they are decreased to normal levels in the late stage of dcSSc characterized by the improvement of skin sclerosis [37]. IL-4 remains at normal levels in the early stage of dcSSc, but it is decreased along with the resolution of skin sclerosis. In contrast, serum IL-12 levels are decreased in the early stage of dcSSc, then they gradually increase in parallel with disease duration and finally reach significantly higher than levels normal controls in the late stage of dcSSc [38]. Thus, Th2-skewed immune polarization is 
quite evident in the early inflammatory phase of dcSSc. Because IL-4 and IL-13 have a profibrotic effect on dermal fibroblasts, such as the induction of type I collagen expression [39], the predominance of Th2 cytokines seems to be involved in the development of tissue fibrosis in SSc. Therefore, Th2-like Tregs may augment BLM-induced dermal fibrosis in $\mathrm{Fli1}^{+/-}$mice. In addition, Th2-like Tregs have the potential to induce SSc-like features in other types of cells under a Fli1-deficient condition. For instance, $\mathrm{Flil}^{+/-}$macrophages preferentially differentiate into an M2 phenotype in response to IL-4 stimulation [13]. In endothelial cells, IL-4 suppresses VE-cadherin expression and reduces vascular integrity [40], which is a characteristic feature of SSc vasculopathy. Because Fli1 deficiency downregulates VE-cadherin expression [14], Th2-like Tregs further promote the induction of SSc-like vascular features in BLM-treated $\mathrm{Fli1}^{+/-}$mice. Also, Fli1 +/- dermal fibroblasts overproduce progranulin, which suppresses the antifibrotic effect of TNF- $\alpha$ [36]. Therefore, dermal fibroblasts preferentially respond to the profibrotic stimuli in BLM-treated $\mathrm{Fli1}^{+/-}$mice. Thus, Th2-like Tregs possibly induced by IL-33-producing dermal fibroblasts coordinately amplify tissue fibrosis through the phenotypical alteration of various kinds of cells in BLM-treated Fli1 ${ }^{+/-}$mice.

In contrast to a significant contribution of IL-33 to Fli1 +/- dermal fibroblast-dependent Th2-like Treg transdifferentiation, there was just a trend toward the induction of Th17-like Tregs by coculture with $\mathrm{Fli}^{+/-}$dermal fibroblasts and the suppression of $\mathrm{Fli}^{+/-}$dermal fibroblastdependent Th17-like Treg transdifferentiation by anti-IL6 antibody. These results suggest that IL- 6 produced by dermal fibroblasts modestly and partially contributes to Th17-like Treg transdifferentiation. Indeed, the fold induction of Fli1 haploinsufficiency-dependent expression was much smaller in IL-6 ( 1.6 times) than in IL-33 $(\sim 33$ times) in dermal fibroblasts. Taken together with the evidence that IL-6 is overproduced by a variety of cells under inflammatory conditions, including endothelial cells, keratinocytes, and inflammatory cells as well as dermal fibroblasts $[41,42]$, the contribution of dermal fibroblasts to the total amount of IL- 6 expression seems to be relatively small in the lesional skin of BLM-treated $\mathrm{Fli}^{+/-}$mice, suggesting a weak contribution of dermal fibroblasts to Th17-like Treg differentiation. However, we need to be aware that IL-6-dependent redifferentiation of Tregs into Th17 cells is dependent on IL- 1 and TGF- $\beta$ [43]. Given that IL-1 $\beta$ is upregulated in the lesional skin of BLM-treated Fli1 ${ }^{+/-}$mice [13] and that integrin $\alpha_{v} \beta_{3}$ and $\alpha_{\mathrm{v}} \beta_{5}$, which promote the release of active TGF- $\beta$ from its latent form $[44,45]$, are upregulated in dermal fibroblasts of BLM-treated $\mathrm{Fli1}^{+/-}$mice [13], IL-6 seems to coordinately promote the differentiation of Th17-like Tregs together with IL-1 $\beta$ and TGF- $\beta$ in the lesional skin of these mice. Therefore, the contribution of fibroblastderived IL-6 to the development of Th17-like Tregs was likely underestimated in the coculture experiment. Another important point is that, in addition to skin-localized transdifferentiation of Th17-like Tregs, the increased infiltration of circulating Th17-like Tregs into the lesional skin may occur in BLM-treated Fli1 ${ }^{+/-}$mice. Relevant to this hypothesis is our previous demonstration that Fli1 deficiency induces the expression of intercellular adhesion molecule-1 and glycosylation-dependent cell adhesion molecule-1, positive regulators for Th2 and Th17 cell infiltration, while suppressing the expression of E-selectin and P-selectin, positive regulators of Th1 cell infiltration $[13,46]$. These previous data propose the possibility that the infiltration of circulating Th17-like Tregs into the lesional skin may be facilitated by altered expression of cell adhesion molecules in BLM-treated $\mathrm{Flil}^{+-}$mice. Further studies are required to clarify these points.

The contribution of IL-17A to the development of skin fibrosis has been well studied in BLM-treated mice. For instance, Th17 cell infiltration and IL-17A expression in the skin are increased and serum IL-17A levels correlate with the severity of skin fibrosis in this murine model [47]. Furthermore, loss of $I l 17 a$, but not Ifny and Il4, results in the reduction of BLM-induced skin fibrosis and IL-17A stimulation induces the expression of TGF- $\beta$ and connective tissue growth factor in NIH3T6 fibroblasts [24]. Therefore, IL-17A serves as a potent profibrotic cytokine in skin fibrosis of BLM-treated mice. According to the present data, we speculate that Fli1 haploinsufficiency enhances Th17-like cell proportions in skinhoming Tregs, subsequently amplifying tissue fibrosis through the profibrotic effect of IL-17A on dermal fibroblasts. Given that $\mathrm{Fli1}^{+/-}$dermal fibroblasts produce excessive amounts of type I collagen under a physiological condition [11], Fli1 haploinsufficiency likely contributes to a feedforward relationship between dermal fibroblasts and Th17-like Tregs, which underlies extensive skin fibrosis.

In a recent study, MacDonald et al. [2] reported that the proportions of conventional Th17 cells in CD4 ${ }^{+} \mathrm{FoxP}^{-} \mathrm{T}$ cells and Th17-like Tregs in $\mathrm{CD}^{+}{ }^{+} \mathrm{FoxP}^{+} \mathrm{T}$ cells are comparable in the skin of 36 patients with SSc (average disease duration $5.7 \pm 1.1$ years for dcSSc and $5.7 \pm 1.8$ years for limited cutaneous systemic sclerosis) and 20 healthy control subjects. In contrast, a significantly increased proportion of Th17-like Tregs in the skin of BLM-treated $\mathrm{Fli1}^{+/-}$mice was evident in the present study. This discrepancy seems to be plausible, considering that the activity and severity of profibrotic inflammation are quite variable in the lesional skin of individual patients with SSc owing to the diverse heterogeneity of this disease, even though patients with similar clinical backgrounds are selected. According to a couple 
of previous reports, the expression of Th17 cytokines is elevated in the lesional skin of patients with early SSc and positively correlates with the severity of skin sclerosis $[25,48-50]$. The reproducibility of these results among several independent studies strongly indicates a critical role of Th17 cytokines in skin fibrosis of SSc. Taking into account the present data indicating that Fli1 haploinsufficiency increases the proportion of Th17-like Tregs under profibrotic inflammatory conditions, Th17like Tregs are potentially involved in the development of skin fibrosis to variable degrees in individual SSc cases.

\section{Conclusions}

To our knowledge, this is the first report demonstrating that Fli1 haploinsufficiency increases Th2- and Th17-like Treg proportions in BLM-induced profibrotic skin condition, in which IL-33-producing dermal fibroblasts contribute to Th2-like Treg transdifferentiation. The present findings indicate that IL-33-dependent transdifferentiation of Th2-like Tregs in SSc lesional skin, a notion proposed by MacDonald et al. [2], is part of an Fli1 deficiencydependent pathological process. Overall, these data further strengthen the importance of Fli1 deficiency in the developmental process of SSc and reinforce the notion that dermal fibroblast-dependent regulation of immune cell phenotypes is involved in the development of skin fibrosis in SSc.

\section{Additional files}

Additional file 1: Figure S1. Scatterplots of Fig. 1b. Scatterplots of the proportions of IL-4-, IL-13-, IL17A-, and IFN- - -producing splenic Tregs from bleomycin (BLM)-treated wild-type and Fli ${ }^{+/-}$mice $(n=6)$. (PDF $\left.592 \mathrm{~kb}\right)$

Additional file 2: Figure S2. Signal intensity of IL-33. Signal intensity of IL-33 was analyzed and summarized. To quantify signal intensity of IL-33, color images were converted to grayscale, and then the brightness was measured in five different randomly selected fibroblasts and epidermal areas per specimen. WT Wild-type mice; Basal Under physiological condition; BLM Bleomycin-treated. (PDF $57 \mathrm{~kb}$ )

Additional file 3: Figure S3. Original films of immunoblotting with molecular weight markers. Original films of Figs. 2e and 4a. Molecular weight markers are shown. (PDF $573 \mathrm{~kb}$ )

\section{Abbreviations \\ AU: Arbitrary units; BLM: Bleomycin; ChIP: Chromatin immunoprecipitation; dcSSc: Diffuse cutaneous systemic sclerosis; Fli1: Friend leukemia virus integration 1; IFN: Interferon; IgG: Immunoglobulin G; IL: Interleukin; MFI: Mean fluorescence intensity; mRNA: Messenger RNA; Treg: Regulatory $T$ cell; SSc: Systemic sclerosis; SSC-A: Side scatter area; TGF- $\beta$ : Transforming growth factor- $\beta$; Th: T helper cell; TNF-a: Tumor necrosis factor-a; WT: Wild type}

\section{Acknowledgements}

We thank Tamami Kaga, Yumi Hasegawa, Satoko Itakura, and Naoko Watanabe for technical assistance.

\section{Funding}

Funding for this work was provided as a project grant for research on intractable diseases from the Ministry of Health, Labour, and Welfare of Japan (16H05366).
Availability of data and materials

Not applicable.

\section{Authors' contributions}

RS and YA conceived of and designed the study. RS, KN, MH, SM, TY, TTan, YI, TTak, TToy, and AY acquired data. RS, YA, KN, MH, SM, TY, TTan, YI, TTak, TToy, AY, MT, and SS analyzed and interpreted data. YA, AY, and SS obtained funding. YA, MT, and SS supervised the study. All authors read and approved the final manuscript.

\section{Ethics approval and consent to participate}

This study was approved by the ethics committee and the committee on animal experimentation of the University of Tokyo Graduate School of Medicine. Human normal dermal fibroblasts were purchased and used; therefore, consent to participate was not applicable.

\section{Consent for publication}

Not applicable.

\section{Competing interests}

The authors declare that they have no competing interests.

\section{Publisher's Note}

Springer Nature remains neutral with regard to jurisdictional claims in published maps and institutional affiliations.

\section{Author details}

'Department of Dermatology, University of Tokyo Graduate School of Medicine, 7-3-1 Hongo, Bunkyo-ku, Tokyo 113-8655, Japan. ${ }^{2}$ Arthritis Center, Boston University School of Medicine, Boston, MA, USA.

Received: 13 July 2017 Accepted: 19 January 2018

Published online: 07 February 2018

References

1. Asano Y, Sato S. Vasculopathy in scleroderma. Semin Immunopathol. 2015;37(5):489-500.

2. MacDonald KG, Dawson NA, Huang Q, Dunne JV, Levings MK, Broady R. Regulatory $T$ cells produce profibrotic cytokines in the skin of patients with systemic sclerosis. J Allergy Clin Immunol. 2015;135(4):946-55.e9.

3. Antiga E, Quaglino P, Bellandi S, Volpi W, Del Bianco E, Comessatti A, OsellaAbate S, De Simone C, Marzano A, Bernengo MG, et al. Regulatory T cells in the skin lesions and blood of patients with systemic sclerosis and morphoea. Br J Dermatol. 2010;162(5):1056-63.

4. Mathian A, Parizot C, Dorgham K, Trad S, Arnaud L, Larsen M, Miyara M, Hie $\mathrm{M}$, Piette JC, Frances $\mathrm{C}$, et al. Activated and resting regulatory $T$ cell exhaustion concurs with high levels of interleukin-22 expression in systemic sclerosis lesions. Ann Rheum Dis. 2012;71(7):1227-34.

5. Slobodin G, Ahmad MS, Rosner I, Peri R, Rozenbaum M, Kessel A, Toubi E, Odeh M. Regulatory T cells $\left(\mathrm{CD}^{+} \mathrm{CD}^{2} 5^{\text {bright }} \mathrm{FoxP}^{+}\right)$expansion in systemic sclerosis correlates with disease activity and severity. Cell Immunol. 2010;261(2):77-80

6. Klein S, Kretz CC, Ruland V, Stumpf C, Haust M, Hartschuh W, Hartmann M, Enk A, Suri-Payer E, Oberle N, et al. Reduction of regulatory T cells in skin lesions but not in peripheral blood of patients with systemic scleroderma. Ann Rheum Dis. 2011;70(8):1475-81.

7. McClymont SA, Putnam AL, Lee MR, Esensten JH, Liu W, Hulme MA, Hoffmuller U, Baron U, Olek S, Bluestone JA, et al. Plasticity of human regulatory $T$ cells in healthy subjects and patients with type 1 diabetes. J Immunol. 2011;186(7):3918-26.

8. Kleinewietfeld M, Hafler DA. The plasticity of human Treg and Th17 cells and its role in autoimmunity. Semin Immunol. 2013;25(4):305-12.

9. Pesenacker AM, Bending D, Ursu S, Wu Q, Nistala K, Wedderburn LR. CD161 defines the subset of FoxP3 ${ }^{+} \mathrm{T}$ cells capable of producing proinflammatory cytokines. Blood. 2013;121(14):2647-58.

10. Asano Y. Epigenetic suppression of Fli1, a potential predisposing factor in the pathogenesis of systemic sclerosis. Int J Biochem Cell Biol. 2015;67:86-91.

11. Wu KK. Analysis of protein-DNA binding by streptavidin-agarose pulldown. Methods Mol Biol. 2006;338:281-90.

12. Hasegawa M. Biomarkers in systemic sclerosis: their potential to predict clinical courses. J Dermatol. 2016;43(1):29-38. 
13. Taniguchi T, Asano Y, Akamata K, Noda S, Takahashi T, Ichimura Y, Toyama T, Trojanowska M, Sato S. Fibrosis, vascular activation, and immune abnormalities resembling systemic sclerosis in bleomycin-treated Fli-1haploinsufficient mice. Arthritis Rheum. 2015;67(2):517-26.

14. Asano Y, Stawski L, Hant F, Highland K, Silver R, Szalai G, Watson DK, Trojanowska M. Endothelial Fli1 deficiency impairs vascular homeostasis: a role in scleroderma vasculopathy. Am J Pathol. 2010;176(4):1983-98.

15. van Bon L, Affandi AJ, Broen J, Christmann RB, Marijnissen RJ, Stawski L, Farina GA, Stifano G, Mathes AL, Cossu M, et al. Proteome-wide analysis and CXCL4 as a biomarker in systemic sclerosis. N Engl J Med. 2014;370(5):433-43.

16. Romano E, Chora I, Manetti M, Mazzotta C, Rosa I, Bellando-Randone S, Blagojevic J, Soares R, Avouac J, Allanore Y, et al. Decreased expression of neuropilin-1 as a novel key factor contributing to peripheral microvasculopathy and defective angiogenesis in systemic sclerosis. Ann Rheum Dis. 2016;75(8):1541-9.

17. Noda S, Asano Y, Nishimura S, Taniguchi T, Fujiu K, Manabe I, Nakamura K, Yamashita T, Saigusa R, Akamata K, et al. Simultaneous downregulation of KLF5 and Fli1 is a key feature underlying systemic sclerosis. Nat Commun. 2014;5:5797.

18. Akamata K, Asano Y, Yamashita T, Noda S, Taniguchi T, Takahashi T, Ichimura Y, Toyama T, Trojanowska M, Sato S. Endothelin receptor blockade ameliorates vascular fragility in endothelial cell-specific Fli-1-knockout mice by increasing Fli-1 DNA binding ability. Arthritis Rheum. 2015;67(5):1335-44.

19. Saigusa R, Asano Y, Yamashita T, Taniguchi T, Takahashi T, Ichimura Y, Toyama T, Yoshizaki A, Miyagaki T, Sugaya M, et al. Fli1 deficiency contributes to the downregulation of endothelial protein $C$ receptor in systemic sclerosis: a possible role in prothrombotic conditions. $\mathrm{Br} \mathrm{J}$ Dermatol. 2016;174(2):338-47.

20. Moore CB, Allen IC. Primary ear fibroblast derivation from mice. Methods Mol Biol. 2013;1031:65-70.

21. Bouwens EA, Stavenuiter F, Mosnier LO. Mechanisms of anticoagulant and cytoprotective actions of the protein C pathway. J Thromb Haemost. 2013; 11(Suppl 1):242-53.

22. Pociask DA, Chen K, Choi SM, Oury TD, Steele C, Kolls JK. ү $\delta$ T cells attenuate bleomycin-induced fibrosis through the production of CXCL10. Am J Pathol. 2011;178(3):1167-76.

23. Ichimura Y, Asano Y, Akamata K, Takahashi T, Noda S, Taniguchi T, Toyama T, Aozasa N, Sumida H, Kuwano $Y$, et al. Fli1 deficiency contributes to the suppression of endothelial CXCL5 expression in systemic sclerosis. Arch Dermatol Res. 2014;306(4):331-8.

24. Okamoto Y, Hasegawa M, Matsushita T, Hamaguchi Y, Huu DL, Iwakura Y, Fujimoto M, Takehara K. Potential roles of interleukin-17A in the development of skin fibrosis in mice. Arthritis Rheum. 2012;64(11):3726-35.

25. Radstake TR, van Bon L, Broen J, Hussiani A, Hesselstrand R, Wuttge DM, Deng $Y$, Simms R, Lubberts E, Lafyatis R. The pronounced Th17 profile in systemic sclerosis (SSc) together with intracellular expression of TGF $\beta$ and IFNy distinguishes SSc phenotypes. PLoS One. 2009;4(6):e5903.

26. Yamamoto T, Takahashi Y, Takagawa S, Katayama I, Nishioka K. Animal model of sclerotic skin. II. Bleomycin induced scleroderma in genetically mast cell deficient WBB6F1-W/W(V) mice. J Rheumatol. 1999;26(12):2628-34.

27. Yamamoto T, Kuroda M, Nishioka K. Animal model of sclerotic skin. III: Histopathological comparison of bleomycin-induced scleroderma in various mice strains. Arch Dermatol Res. 2000;292(11):535-41.

28. Takagawa S, Lakos G, Mori Y, Yamamoto T, Nishioka K, Varga J. Sustained activation of fibroblast transforming growth factor- $\beta / S$ mad signaling in a murine model of scleroderma. J Invest Dermatol. 2003;121(1):41-50.

29. Seltmann J, Werfel T, Wittmann M. Evidence for a regulatory loop between IFN- $\gamma$ and IL-33 in skin inflammation. Exp Dermatol. 2013;22(2):102-7.

30. Bettelli E, Carrier Y, Gao W, Korn T, Strom TB, Oukka M, Weiner HL, Kuchroo VK. Reciprocal developmental pathways for the generation of pathogenic effector $T_{H} 17$ and regulatory T cells. Nature. 2006;441(7090):235-8.

31. Xu L, Kitani A, Fuss I, Strober W. Cutting edge: regulatory T cells induce CD4 ${ }^{+} \mathrm{CD} 25^{-}$Foxp3 ${ }^{-} \mathrm{T}$ cells or are self-induced to become Th17 cells in the absence of exogenous TGF- $\beta$. J Immunol. 2007;178(11):6725-9.

32. Yamamoto T. Animal model of systemic sclerosis. J Dermatol. 2010;37(1):26-41.

33. Yamamoto T. The bleomycin-induced scleroderma model: what have we learned for scleroderma pathogenesis? Arch Dermatol Res. 2006;297(8):333-44.

34. Korn JH, Mayes M, Matucci Cerinic M, Rainisio M, Pope J, Hachulla E, Rich E, Carpentier P, Molitor J, Seibold JR, et al. Digital ulcers in systemic sclerosis: prevention by treatment with bosentan, an oral endothelin receptor antagonist. Arthritis Rheum. 2004;50(12):3985-93.
35. Czuwara-Ladykowska J, Shirasaki F, Jackers P, Watson DK, Trojanowska M. Fli-1 inhibits collagen type I production in dermal fibroblasts via an Sp1dependent pathway. J Biol Chem. 2001;276(24):20839-48.

36. Ichimura Y, Asano Y, Akamata K, Noda S, Taniquchi T, Takahashi T, Toyama T, Tada Y, Sugaya M, Sato S, et al. Progranulin overproduction due to Fli-1 deficiency contributes to the resistance of dermal fibroblasts to tumor necrosis factor in systemic sclerosis. Arthritis Rheum. 2015;67(12):3245-55.

37. Sato S, Hasegawa M, Takehara K. Serum levels of interleukin- 6 and interleukin-10 correlate with total skin thickness score in patients with systemic sclerosis. J Dermatol Sci. 2001;27(2):140-6.

38. Matsushita T, Hasegawa M, Hamaguchi Y, Takehara K, Sato S. Longitudinal analysis of serum cytokine concentrations in systemic sclerosis: association of interleukin 12 elevation with spontaneous regression of skin sclerosis. J Rheumatol. 2006;33(2):275-84.

39. Bhogal RK, Bona CA. Regulatory effect of extracellular signal-regulated kinases (ERK) on type I collagen synthesis in human dermal fibroblasts stimulated by IL-4 and IL-13. Int Rev Immunol. 2008;27(6):472-96.

40. Chalubinski M, Wojdan K, Luczak E, Gorzelak P, Borowiec M, Gajewski A, Rudnicka K, Chmiela M, Broncel M. IL-33 and IL-4 impair barrier functions of human vascular endothelium via different mechanisms. Vasc Pharmacol. 2015;73:57-63.

41. Khan K, Xu S, Nihtyanova S, Derrett-Smith E, Abraham D, Denton CP, Ong $\mathrm{VH}$. Clinical and pathological significance of interleukin 6 overexpression in systemic sclerosis. Ann Rheum Dis. 2012;71(7):1235-42.

42. Desallais L, Avouac J, Frechet M, Elhai M, Ratsimandresy R, Montes M, Mouhsine H, Do H, Zagury JF, Allanore Y. Targeting IL-6 by both passive or active immunization strategies prevents bleomycin-induced skin fibrosis. Arthritis Res Ther. 2014;16(4):R157.

43. Yang XO, Nurieva R, Martinez GJ, Kang HS, Chung Y, Pappu BP, Shah B, Chang SH, Schluns KS, Watowich SS, et al. Molecular antagonism and plasticity of regulatory and inflammatory $\mathrm{T}$ cell programs. Immunity. 2008;29(1):44-56.

44. Asano $Y$, Ihn H, Yamane K, Jinnin M, Mimura Y, Tamaki K. Increased expression of integrin $a_{v} \beta_{3}$ contributes to the establishment of autocrine TGF- $\beta$ signaling in scleroderma fibroblasts. J Immunol. 2005;175(11):7708-18.

45. Asano $Y$, Ihn H, Yamane K, Jinnin M, Mimura Y, Tamaki K. Involvement of $a_{v} \beta_{5}$ integrin-mediated activation of latent transforming growth factor $\beta 1$ in autocrine transforming growth factor $\beta$ signaling in systemic sclerosis fibroblasts. Arthritis Rheum. 2005;52(9):2897-905.

46. Yoshizaki A, Yanaba K, Iwata Y, Komura K, Ogawa A, Akiyama Y, Muroi E, Hara T, Ogawa F, Takenaka $M$, et al. Cell adhesion molecules regulate fibrotic process via Th1/Th2/Th17 cell balance in a bleomycin-induced scleroderma model. J Immunol. 2010;185(4):2502-15.

47. Lei L, Zhao C, Qin F, He ZY, Wang X, Zhong XN. Th17 cells and IL-17 promote the skin and lung inflammation and fibrosis process in a bleomycin-induced murine model of systemic sclerosis. Clin Exp Rheumatol. 2016;34(5 Suppl 100):14-22.

48. Zhou Y, Hou W, Xu K, Han D, Jiang C, Mou K, Li Y, Meng L, Lu S. The elevated expression of Th17-related cytokines and receptors is associated with skin lesion severity in early systemic sclerosis. Hum Immunol. 2015;76(1):22-9.

49. Yang $X$, Yang J, Xing X, Wan L, Li M. Increased frequency of Th17 cells in systemic sclerosis is related to disease activity and collagen overproduction. Arthritis Res Ther. 2014;16(1):R4

50. Murata M, Fujimoto M, Matsushita $T$, Hamaguchi $Y$, Hasegawa M, Takehara K, Komura K, Sato S. Clinical association of serum interleukin17 levels in systemic sclerosis: is systemic sclerosis a Th17 disease? J Dermatol Sci. 2008;50(3):240-2. 